\title{
Metabolic profiling of S-praziquantel: Structure elucidation using the crystalline sponge method in combination with mass spectrometry and nuclear magnetic resonance
}

Lara Rosenberger, Judith Jenniches, Carolina von Essen, Anupam Khutia, Clemens Kühn, Andreas Marx, Katrin Georgi, Anna K. H. Hirsch, Rolf W. Hartmann, Lassina Badolo

Merck KGaA, Frankfurter Strasse 250, 64293 Darmstadt, Germany (L.R., J.J., C.v.E., A.K., C.K., A.M., K.G., L.B.)

Helmholtz-Institute for Pharmaceutical Research Saarland (HIPS) - Helmholtz Centre for Infection Research (HZI), Campus E8.1, 66123 Saarbrücken, Germany (L.R., A.K.H.H, R.W.H.)

Department of Pharmacy, Saarland University, Campus E8.1, 66123 Saarbrücken, Germany (L.R., A.K.H.H, R.W.H.) 
Running Title: Structural elucidation of metabolites by crystalline sponges

\section{Address correspondence to:}

Lara Rosenberger,

Merck KGaA,

Frankfurter Str. 250, 64293 Darmstadt, Germany

E-mail: Lara.Rosenberger@emdgroup.com

Number of Text Pages: 21

Number of Tables: 0

Number of Figures: 5

Number of References: 26

Number of words in Abstract: 250

Number of words in Introduction: 484

Number of words in Discussion: 688

Abbreviations: API, active pharmaceutical ingredient; CS, crystalline sponge; CS-XRD, crystalline sponge method; DME, 1,2-dimethoxyethane; DMSO- $d_{6}$, dimethyl sulfoxide- $d_{6} ; \mathrm{HLM}$, human liver microsomes; HPLC, high performance liquid chromatography; LC, liquid chromatography; MS, mass spectrometry; PZQ, praziquantel; qTOF, quadrupole time-of-flight mass spectrometry; $R_{1}$, residual factor; $R_{\text {int, }}$ internal $R$-value; ROESY, rotating frame Overhauser enhancement effect spectroscopy; SC-XRD, single-crystal X-ray diffraction; tpt, 2,4,6-tris(4-pyridyl)-1,3,5-triazine; UPLC, ultra-performance liquid chromatography 


\section{Abstract}

Praziquantel (PZQ) is the drug of choice for treatment of the neglected tropical disease schistosomiasis. Although the drug has been extensively used over several decades and its metabolism well studied (several oxidative metabolites are known from literature), the knowledge of the complete structure of some of its metabolites remains elusive. Conventional techniques like nuclear magnetic resonance (NMR) or liquid chromatography mass spectrometry (LC-MS) were used in the past to investigate phase I and phase II metabolites of PZQ. These techniques are either limited to provide the complete molecular structure (LCMS) or require large amount of sample material (NMR), which are not always available when in vitro systems are used for investigation of the metabolites. In this study, we describe new structures of $S-P Z Q$ metabolites generated in vitro from human liver microsomes (HLM) using the crystalline sponge method (CS-XRD). After chromatographic separation and purification of the oxidative metabolites, ultra-performance liquid chromatography (UPLC)-quadrupole timeof-flight mass spectrometry (qTOF) analysis was conducted to narrow down the position of oxidation to a certain part of the molecule. To determine the exact position of hydroxylation, singe-crystal X-ray diffraction (SC-XRD) analysis of the crystalline sponges (CS) and absorbed analyte was used to identify the structure of S-PZQ and its metabolites. CS-XRD allowed for complete structure elucidation of the known metabolites S-trans-4'-hydroxy PZQ (M1), S-cis4'-hydroxy PZQ (M2) and S-/R-11b-hydroxy PZQ (M6) as well as the unknown metabolites S9-hydroxy PZQ (M3) and S-7-hydroxy S-PZQ (M4). For comparison of structural elucidation techniques, one metabolite (M3) was additionally analyzed using NMR. 


\section{Significance Statement}

The information content of the metabolic pathway of praziquantel is still limited. The crystalline sponge method allowed the complete structural elucidation of three known and two unknown metabolites of $S$-praziquantel, using only trace amounts of analyte material, as demonstrated in this study. 


\section{Introduction}

Schistosomiasis is a neglected tropical disease caused by a parasitic flatworm of the genus Schistosoma and affects, with a high prevalence in Africa, almost 240 million people worldwide. The World Health Organization implemented a program for schistosomiasis control over the past 40 years and recommends the anthelmintic drug PZQ as treatment of choice for all forms of the disease (WHO, 2011, 2020). PZQ is currently used as a racemic formulation in therapy, however, the activity is mainly associated with the R-enantiomer (Meister et al., 2014; Kovač et al., 2017). Although the drug has been known and extensively used since the early 1980s, the information content of the metabolic pathway is limited. Enantioselective transformation of $R-P Z Q$ and $S-P Z Q$ has been explored in various studies, showing different metabolic profiles for both enantiomers (Wang et al., 2014; Vendrell-Navarro et al., 2020, Park et al., 2021). Several mono-oxidized (+16 Da) and secondary oxidative metabolites (+32 and +14 Da) are known from the literature but most of their complete structures are still unknown. The main metabolite in human is 4'-hydroxy PZQ and the position of hydroxylation was identified both in cis and trans configuration (Nleya et al., 2019). Two more metabolites have been described as 8-hydroxy PZQ and 11b-hydroxy PZQ by isolation from human urine and in vitro recombinant human P450 reactions, using NMR for data analysis (Schepmann and Blaschke, 2001; Vendrell-Navarro et al., 2020). Additional metabolites have been analyzed using different LCMS systems, giving information of the type of metabolism (i.e., hydroxylation, dehydrogenation and glucuronidation) and allowing to narrow down the site of metabolism to a certain part of the molecule (Lerch and Blaschke, 1998; Huang et al., 2010; Wang et al., 2014). Despite the advantage of LC-MS techniques to reach high sensitivity for sample analysis from in vitro origin, these techniques failed to identify the complete structure of metabolites.

A new approach for structural elucidation of compounds was introduced in 2013 by Makoto Fujita and is commonly known as "crystalline sponge method" (CS-XRD) (Inokuma et al., 2013). The method enables crystal structure determination without the limitation of crystallization with only nanogram to a few microgram of analyte material. A metal coordination 
complex $\left[\left(\mathrm{ZnX}_{2}\right)_{3} \cdot(\mathrm{tpt})_{2}\right]_{\mathrm{n}} \cdot \mathrm{X}(\text { solvent })_{\mathrm{m}}(\mathrm{X}=\mathrm{I} ; \mathrm{tpt}=2,4,6$-tris (4-pyridyl)-1,3,5-triazine) is used as a pre-existing crystal and functions as a host. During a "soaking process", the analyte (guest) is absorbed into the pores of the three-dimensional framework via diffusion and regularly ordered by intermolecular, noncovalent interactions and thus accessible for X-ray diffraction analysis (Inokuma et al., 2016; Brunet et al., 2017; Sakurai et al., 2017). We recently demonstrated that the CS-XRD is a valuable tool, which can be applied to identify the exact position of metabolism for phase I and phase II metabolites generated in low amounts from in vitro incubation (Rosenberger et al., 2020).

In this study, we now apply the CS-XRD for the determination of the absolute structures of $S$ PZQ and six of its hydroxylated metabolites, after incubation with HLM. 


\section{Materials and Methods}

Chemicals and Reagents. S-PZQ, S-cis-4'-hydroxy PZQ and S-trans-4'-hydroxy PZQ were obtained from Merck KGaA small molecule library. Dipotassium hydrogen phosphate, potassium dihydrogen phosphate, magnesium chloride hexahydrate, cyclohexane, n-hexane, dihydronicotinamide adenine dinucleotide phosphate tetrasodium salt (NADPH-Na4), dimethyl sulfoxide- $d_{6}\left(\right.$ DMSO- $\left.d_{6}\right)$, water (UHPLC-MS grade) and acetonitrile (UHPLC-MS grade) were purchased from Merck KGaA (Darmstadt, Germany). Zinc iodide, 1,2-dimethoxyethane (DME) and formic acid were purchased from Sigma Aldrich Chemie GmbH (Steinheim, Germany). 2,4,6-tri(4-pyridyl)-1,3,5-triazine (tpt) was purchased from abcr GmbH (Karlsruhe, Germany). Mixed gender HLM (Ultrapool, pool of 150) were purchased from Corning (Corning, USA).

Metabolism of S-PZQ by HLM. The hydroxylation of S-PZQ was conducted with an incubation mixture containing $0.5 \mathrm{mg} / \mathrm{ml} \mathrm{HLM}, 50 \mathrm{mM}$ potassium phosphate buffer $(\mathrm{pH} 7.4), 1 \mathrm{mM}$ magnesium chloride and $200 \mu \mathrm{M}$ substrate. After 5 min of preincubation $\left(37^{\circ} \mathrm{C}, 150 \mathrm{rpm}\right)$, the reaction was initiated by the addition of $\mathrm{NADPH}(1.5 \mathrm{mM})$, and the mixture was incubated for another $24 \mathrm{~h}$ (final volume: $5.40 \mathrm{ml}$ ). After $6 \mathrm{~h}$, the reaction was boosted by addition of NADPH $(1.5 \mathrm{mM})$.

The reaction was quenched by adding one volume of ice-cold acetonitrile and the precipitated proteins were removed by centrifugation at $4000 \mathrm{~g}$ for $1 \mathrm{~h}$ at $4{ }^{\circ} \mathrm{C}$. The supernatant was evaporated to dryness (nitrogen flow, $40^{\circ} \mathrm{C}$ ) and resolubilized in acetonitrile/water (20:80). 100 $\mu \mathrm{l}$ aliquots were used for purification and fractionation by high performance liquid chromatography coupled with mass spectrometry (HPLC-MS).

UPLC-qTOF. The supernatants were analyzed on a Waters Acquity UPLC system combined with a Xevo G2-S quadrupole time-of-flight mass spectrometer (qTOF) (Waters Corporation). Analysis was performed with an electrospray ion source in positive ion mode. For MS scan, the QToF was operating with a source temperature at $150{ }^{\circ} \mathrm{C}$, a desolvation temperature at $600^{\circ} \mathrm{C}$ and a capillary voltage at $0.5 \mathrm{kV}$. For full-scan MS mode, the collision energy was set 
to $4 \mathrm{~V}$ and the mass range was set to $\mathrm{m} / \mathrm{z} 100$ to 1000 . For MS/MS studies, QToF MS acquisition was conducted using a collision energy ranging from 25 to $40 \mathrm{~V}$.

Metabolite separation was achieved on a chiral Lux Cellulose-2 column (150-2 mm, 3 Hm; Phenomenex) equipped with a Lux Cellulose-2 precolumn (3 $\mathrm{mm}$ inner diameter; Phenomenex) at a flow rate of $0.45 \mathrm{ml} / \mathrm{min}$ over a period of $20 \mathrm{~min}$ using solvent $\mathrm{A}$ (water + $0.1 \%$ formic acid) and solvent $B$ (acetonitrile $+0.1 \%$ formic acid) as mobile phases. The column oven temperature was set to $40{ }^{\circ} \mathrm{C}$. Elution was performed using a gradient from $20 \%$ to $90 \%$ $\mathrm{B}$ in $15 \mathrm{~min}, 90 \%$ to $95 \% \mathrm{~B}$ in $0.2 \mathrm{~min}, 95 \% \mathrm{~B}$ for $2.1 \mathrm{~min}$, returning to $20 \% \mathrm{~B}$ in $0.1 \mathrm{~min}$ and re-equilibration at 20\% B for $2.6 \mathrm{~min}$. The software UNIFI version 1.9.4 (Waters Corporation) was used to support the identification of metabolites. The metabolites were evaluated for mass error $(\leq 4 \mathrm{ppm})$, fragmentation pattern and retention time.

HPLC-MS. The supernatants were analyzed and fractionated on an Acquity Arc HPLC system (Waters Corporation). The equipment components were described in detail in our previous work (Rosenberger et al., 2020).

Samples were analyzed with electrospray ionization mass spectrometry in positive ionization mode within an acquisition range from $\mathrm{m} / \mathrm{z} 100$ to $\mathrm{m} / \mathrm{z} 650$ in continuum mode and a sampling frequency of $2 \mathrm{~Hz}$. The capillary voltage and the cone voltage were set to $0.8 \mathrm{~V}$ and $10.0 \mathrm{~V}$, respectively. The probe temperature was set to $600{ }^{\circ} \mathrm{C}$. The mass value of $\mathrm{m} / \mathrm{z} 329$ was registered by the MS detector and triggered the fractionation of the target analytes. The software MassLynx 4.2 and FractionLynx were used for data acquisition and sample fractionation.

HPLC separation for M4 and M6 PZQ was performed with two Chromolith Performance RP18e columns (100-4.6 mm; Merck KGaA) for primary chromatographic purification and one Purospher Star RP18e Hibar HR column (100-2.1 mm, $2 \mu \mathrm{m}$; Merck KGaA) as second chromatographic system. The column oven temperature was set to $25{ }^{\circ} \mathrm{C}$. For the first purification, elution was performed at a flow rate of $1.0 \mathrm{ml} / \mathrm{min}$ using solvent $A$ (water) and solvent B (acetonitrile) as mobile phases. The analytes were eluted over a period of $30 \mathrm{~min}$, 
using a multi-segmented gradient from $0 \%$ to $25 \% \mathrm{~B}$ in $7 \mathrm{~min}, 25 \%$ to $30 \% \mathrm{~B}$ in $3 \mathrm{~min}, 30 \%$ to $80 \% \mathrm{~B}$ in $10 \mathrm{~min}, 80 \% \mathrm{~B}$ for $5 \mathrm{~min}$, returning to $0 \% \mathrm{~B}$ in $0.1 \mathrm{~min}$ and re-equilibration at $0 \% \mathrm{~B}$ for $4.9 \mathrm{~min}$. The eluent was directed to the waste during the first $5 \mathrm{~min}$ to reduce contamination. For the second purification, elution was performed at a flow rate of $0.45 \mathrm{ml} / \mathrm{min}$ over a period of $15 \mathrm{~min}$, applying the following gradient: mobile phase $0 \%$ to $60 \%$ for $10 \mathrm{~min}, 60 \% \mathrm{~B}$ to $100 \%$ $\mathrm{B}$ in $2 \mathrm{~min} ; 100 \%$ to $0 \% \mathrm{~B}$ in $0.1 \mathrm{~min}$ and re-equilibration at $0 \% \mathrm{~B}$ for $2.9 \mathrm{~min}$.

HPLC separation for M3, M5 and additionally M6 PZQ was achieved using an additional third purification step. The further purification was performed using the Lux Cellulose-2 column and gradient described as before. Differences were the column oven temperature of $25^{\circ} \mathrm{C}$ and the solvent $A$ (water) and solvent $B$ (acetonitrile) as mobile phases.

Following each chromatographic separation step, the collected fractions were pooled, evaporated to dryness at $40^{\circ} \mathrm{C}$ under nitrogen flow and resolubilized in acetonitrile/water $(20: 80)$ for further purification. After final purification, the collected fractions from each metabolite were combined and evaporated to dryness to conduct the CS soaking and NMR measurement.

CS-XRD. The porous CS $\left[\left(\mathrm{ZnI}_{2}\right)_{3} \cdot(\mathrm{tpt})_{2}\right]_{\mathrm{n}} \cdot x(\text { cyclohexane })_{\mathrm{m}}(\mathbf{1 a})$ and $\left[\left(\mathrm{Znl_{2 }}\right)_{3} \cdot(\mathrm{tpt})_{2}\right]_{\mathrm{n}} \cdot \mathrm{x}(\mathrm{n}-$ hexane $)_{m}(\mathbf{1 b})$ were prepared according to the procedures known from literature (Biradha and Fujita, 2002; Ramadhar et al., 2015).

For guest soaking of S-PZQ one single crystal of 1 a was transferred with $50 \mu$ cyclohexane to a glass vial. After addition of $2 \mu \mathrm{l}$ of dissolved analyte solution ( $1 \mathrm{mg} / \mathrm{ml}$ in DME), the sample vial was closed with a screw cap and the septum seal was pierced with a syringe needle, before placing it in an incubator at $50{ }^{\circ} \mathrm{C}$ for 1 day. Following evaporation of solvent, the vial was placed without a needle for 2 days at $4{ }^{\circ} \mathrm{C}$ to increase guest occupancy and hence the chirality transfer from the guest molecules to the CS framework. For S-trans-4'-hydroxy PZQ, one crystal of $\mathbf{1 b}$ was used and analyzed after evaporation of $n$-hexane after 1 day. The soaking condition for S-cis-4'-hydroxy PZQ was adjusted by reducing the volume of $n$-hexane to $25 \mu \mathrm{l}$, the temperature for solvent evaporation to $25^{\circ} \mathrm{C}$ and the soaking time to 3 days by 
using a syringe needle with smaller diameter. Soaking of incubation samples (about $1-2 \mu \mathrm{g}$ ) was conducted similarly to the reference material. $2 \mu \mathrm{l}$ of DME, $50 \mu \mathrm{l}$ cyclohexane and one CS (1a) were pipetted into the vial, which contained the pooled metabolite material. The incubator temperature was set to $50{ }^{\circ} \mathrm{C}$ and the evaporation time was one day.

SC-XRD measurements were performed by using a Rigaku Oxford Diffraction XtaLAB Synergy-R diffractometer $\left(\mathrm{Cu}-\mathrm{K}_{\alpha}, \lambda=1.54184 \AA\right)$ at a temperature of $100 \mathrm{~K}$ and crystal structure modeling using OLEX2 (Dolomanov et al., 2009), SHELXT and SHELXL (Sheldrick, 2015) were conducted as described in detail in our previous work (Rosenberger et al., 2020). The software ShelXle was used to generate the electron density maps of S-PZQ and its hydroxy metabolites (Hübschle et al., 2011).

NMR of PZQ Metabolite M3. Structural elucidation of metabolite M3 was performed at $298 \mathrm{~K}$ using a $700 \mathrm{MHz}$ Bruker Avance III equipped with a 5-mm cryocooled triple resonance probe. The isolated metabolite (about $15 \mu \mathrm{g}$ ) was dissolved in $200 \mu \mathrm{LMSO}-d_{6}$ and transferred into a $3 \mathrm{~mm}$ NMR tube.

The ${ }^{1} \mathrm{H}$ NMR spectrum was acquired with $64 \mathrm{k}$ time domain points, a spectral width of $20 \mathrm{ppm}$, a relaxation delay of 10 seconds, and 1024 scans. Edited heteronuclear single quantum coherence NMR spectrum was recorded with 1024 × 256 time domain data points over a spectral width of $12 \mathrm{ppm}$ in the t2 and $165 \mathrm{ppm}$ in the $\mathrm{t} 1$ dimension. The homonuclear correlation spectroscopy NMR spectrum was acquired with 1024 × 256 time domain data points over a spectral width of $12 \mathrm{ppm}$ in the $\mathrm{t} 2$ and $\mathrm{t} 1$ dimension. The rotating frame Overhauser enhancement effect spectroscopy (ROESY) NMR spectrum was recorded with 1024 x 256 time domain data points over a spectral width of $12 \mathrm{ppm}$ in the $\mathrm{t} 2$ and $\mathrm{t} 1$ dimension. The software MestReNova version 14.1.0 (Mestrelab Research) was used for data analysis. 


\section{Results}

UPLC-qTOF analysis of S-PZQ metabolites prepared by incubation with HLM. Both enantiomers of PZQ (Figure 1) were incubated with HLM for 24 h respectively, for metabolite profiling and identification (data not shown). S-PZQ showed an increased formation of metabolites and was therefore chosen for further analysis. During the study of metabolic stability, the UPLC-qTOF chromatogram of S-PZQ, incubated with HLM in the presence of $\mathrm{NADPH}$, showed the formation of several oxidative metabolites $(+16,+32$ and $+14 \mathrm{Da})$, which is in line with already published data (Huang et al., 2010; Wang et al., 2014). The incubation of S-PZQ without cofactor was conducted in parallel and showed no formation of metabolites, indicating their generation by enzymatic reaction. The analysis presented in this study, however, focuses on six monohydroxylated metabolites of S-PZQ (M1-M6).

The metabolites $M 1-M 6$ were detected at a protonated molecular mass $[M+H]^{+}$of $m / z 329$. The increased $m / z$ value of $16 \mathrm{Da}$, compared to the $[\mathrm{M}+\mathrm{H}]^{+}$ion of the parent $(\mathrm{m} / \mathrm{z} 313)$, suggested the addition of one oxygen to the molecular scaffold.

The mono-oxidized metabolites were further analyzed based on their MS/MS spectra and accurate mass measurements (Figure 2, Supplemental Table 1). The mass spectrum $\left([\mathrm{M}+\mathrm{H}]^{+}\right)$ of $\mathrm{M} 1$ and $\mathrm{M} 2$ showed similar fragment ion peaks as the parent compound. The main fragment ions at $m / z 203$ and $m / z 174$ were assigned to the "core moiety" ((i.e., hexahydro-pyrazino $[2,1$-a]isoquinolin-4-one), indicating that the oxidation occurred at the cyclohexane ring. Metabolites M1 and M2 were identified as S-trans-4'-hydroxy PZQ and S-cis-4'-hydroxy PZQ by comparison of the respective retention time with the reference material.

The main fragment ions at $m / z 219, \mathrm{~m} / \mathrm{z} 190, \mathrm{~m} / \mathrm{z} 162$ and $\mathrm{m} / \mathrm{z} 148$, observed in the spectra of both M3 and M5, were assigned to an oxidized core moiety, indicating that the hydroxylation occurred somewhere at the tetrahydro-isoquinoline ring system. The full-scan mass spectrum of metabolites M4 and M6 showed the abundant ion $\mathrm{m} / \mathrm{z} 311$, representing a predominant loss of water (329 -> 311). In addition, both metabolites fragmented to form characteristic ions of $m / z 201, m / z 144$ and $m / z 130$, indicating a spontaneous water loss of the fragment ions observed for M3 and M5. The loss of water is predominantly observed for aliphatic 
hydroxylation, whereas it is less common for hydroxylation reactions, which occur on phenyl rings (Ramanathan et al., 2000; Holčapek et al., 2010). Therefore, it is likely that the addition of one oxygen occurred in the aliphatic part of the core moiety, and not on the aromatic benzene.

The MS/MS fragmentation pattern allowed to limit the oxidation to a certain part of the molecule but could not reveal the actual positions of hydroxylation. The metabolite structures of S-PZQ were therefore further analyzed using the CS-XRD and NMR.

Structural elucidation of S-PZQ metabolites (M1-M4 and M6) by CS-XRD. For successful structure determination using the CS-XRD, the optimization of soaking conditions (e.g., CS type, solvent, temperature, time) is the crucial step. Thereby, various parameters need to be examined for every analyte (Hoshino et al., 2016). The optimal soaking parameters for S-PZQ were selected by conducting a CS affinity screening and examining the affinity of target analyte to CS framework using two CS types $\left[\left(\mathrm{ZnI}_{2}\right)_{3} \cdot(\mathrm{tpt})_{2}\right]_{\mathrm{n}} \cdot \mathrm{x}(\text { solvent })_{\mathrm{m}}$ and $\left[\left(\mathrm{ZnCl}_{2}\right)_{3} \cdot(\mathrm{tpt})_{2}\right]_{\mathrm{n}} \cdot \mathrm{x}(\text { solvent })_{\mathrm{m}} ;$ solvent=cyclohexane, $n$-hexane $)$ at three different temperatures $\left(50^{\circ} \mathrm{C}, 25^{\circ} \mathrm{C}, 4^{\circ} \mathrm{C}\right)$ (data not shown). The screening method has been described in detail in our previous work (Rosenberger et al., 2021). The optimal soaking conditions were then applied for S-PZQ and its mono-oxidized metabolites. To increase the occupancy of the analyte in the CS pores and to maximize the intermolecular interactions between CS framework and guest molecules, the soaking time was further adjusted for some analytes.

The parent API and the reference metabolites S-trans-4'-hydroxy PZQ (M1) and S-cis-4'hydroxy PZQ (M2) could successfully be structurally elucidated with the CS-XRD using a conventional X-ray diffractometer. The crystal structure of S-PZQ revealed one guest molecule with an occupancy of $64 \%$ and one additional cyclohexane molecule with final $R_{1}$ and $R_{\text {int }}$ values of $8.23 \%$ and $3.08 \%$, respectively. A Flack parameter of $0.234(8)$ was obtained, suggesting that the absolute configuration $\mathrm{PZQ}$ is $S$. One molecule of metabolite M1 was assigned by its electron density in the asymmetric unit with an occupancy of $52 \%\left(R_{1} 6.58 \%\right.$ and $\left.\mathrm{R}_{\text {int }} 3.86 \%, \mathrm{X}=0.229(8)\right)$. First analysis of the reference material $\mathrm{M} 2$ resulted in the 
determination of the hydroxy group at the 4'-cyclohexyl moiety in trans configuration. Pharmacokinetic data from previous studies showed the preferred formation of M1 and a cis to trans interconversion (Nleya et al., 2019; Vendrell-Navarro et al., 2020). To influence the less favored axial position of the substituent as little as possible, the soaking process was further optimized by decreasing the temperature to $25^{\circ} \mathrm{C}$. As a result, one guest molecule in cis configuration could be assigned to $M 2$ (occupancy $54 \%, R_{1} 5.85 \%, R_{\text {int }} 2.86 \%, x=$ $0.328(8))$

Next, the CS-XRD was applied to the mono-hydroxylated metabolites of S-PZQ generated by in vitro incubation. The formation of several metabolites in different quantities was observed, necessitating an extensive chromatographic separation and purification process to fractionate the very closely eluting metabolites (Figure 3A). To generate the sample amount in high purity, M4 and M6 were purified using two different chromatographic columns. M3 and M5 were thereby detected as one peak, requiring further separation using a third column (Figure 3B). To estimate the amounts of M3-M6, the MS (for M3 and M5) and UV (for M4 and M6) signals of the reference material M2 were used. Thereby, an increase in the UV-VIS absorption maxima was observed for M3 (278 nm) and M5 (281 nm) compared to S-PZQ or M6 (263 nm), indicating an aromatic hydroxylation at the benzene moiety.

To reveal the exact position of hydroxylation, the soaking experiments were conducted under similar conditions as used for the parent compound to elucidate the structure of metabolites M3-M6. XRD measurements of the soaked CS allowed the crystallographic analysis of the metabolite structures. M6 shows high electron density at position $11 \mathrm{~b}$ of the isoquinoline ring system, confirming the structure elucidated via NMR in previous works (Vendrell-Navarro et al., 2020). High electron density in close proximity to the isoquinoline part was also observed for M4 at position 7 and M3 at position 9. Metabolite M5 shows diffuse electron density in the CS pores, but no structure could be successfully assigned. The electron density map of the "core moiety" from S-PZQ and its successfully elucidated metabolites are shown in Figure 4. Crystallographic data of $M 3$ revealed one analyte molecule with an occupancy of $60 \%\left(R_{1}\right.$ $\left.5.53 \%, R_{\text {int }} 1.52 \%, x=0.204(7)\right)$ and two cyclohexane molecules. 
The crystal structure of M4 revealed one molecule with an occupancy of $76 \%$, three cyclohexane molecules and final $R_{1}$ and $R_{\text {int }}$ values of $4.99 \%$ and $1.45 \%$, respectively. The addition of one oxygen at position 7 of the molecule caused the creation of a second chiral center, which could be determined as $S$ configuration indicated on the Flack parameter value of $0.191(7)$.

As already observed for the other metabolites and the parent compound, M6 was expected to be enantiopure. Surprisingly, an inconclusive Flack parameter of 0.5 , indicating a racemic mixture, was observed. Furthermore, when analyzing the CS pores, both $S$ - and $R-11 \mathrm{~b}$ hydroxy PZQ (occupancy $40 \%$ and $30 \%$ ) were observed $\left(R_{1} 5.92 \%\right.$; $R_{\text {int }} 2.48 \%$ ). The observation was further on confirmed by chiral chromatography with the separation of both enantiomers. After chromatographic separation and purification, both enantiomers were examined separately by CS-XRD. The soaking experiment of the first enantiomer of M6 showed the presence of one $S$-11b-hydroxy PZQ molecule, but due to low occupancy of the analyte and therefore an insufficient chirality transfer from guest molecules to the host framework, the resulting inconclusive Flack value did not allow the confirmation of the chiral center. However, when analyzing the second enantiomer, one molecule of $R-11 \mathrm{~b}$-hydroxy PZQ (occupancy $100 \%$ ) could be observed in the CS pores $\left(R_{1} 7.32 \%\right.$; $R_{\text {int }} 3.69 \%, x=0.221(8)$ ) and hence indicating the identification of the first enantiomer to be in $S$ configuration.

The refined crystallographic structures of $S-P Z Q$ and its hydroxy metabolites are shown in Figure 3. Crystallographic data, ORTEP diagrams of the asymmetric unit including CS framework and analytes, reciprocal ho/ layers and Flack parameters before and after using solvent masking are shown in the Supplemental Data (Supplemental Figure 1-22).

Characterization of M3 by NMR. Additional to the CS-XRD experiments, M3 was further isolated to characterize the structure by NMR analysis (Supplemental Data). The results confirmed the position of hydroxylation at position 9 of the benzene moiety. The following signals were assigned to the structure in the ${ }^{1} \mathrm{H}$ NMR measurement $\left(700 \mathrm{MHz}\right.$, DMSO- $\left.d_{6}\right)$. The NMR spectra indicated the presence of two conformers, which are indicated in the 
following assignment by superscript A and B. The numbering is depicted in Figure 5: $\delta 7.25$ $\left(\mathrm{d}, J=8.3 \mathrm{~Hz}, 1 \mathrm{H}, 11^{\mathrm{A}}\right), 7.05\left(\mathrm{~d}, J=8.5 \mathrm{~Hz}, 1 \mathrm{H}, 11^{\mathrm{B}}\right), 6.72-6.64\left(\mathrm{~m}, 2 \mathrm{H}, 10^{\mathrm{A}, \mathrm{B}}\right), 6.58(\mathrm{~s}, 2 \mathrm{H}$, 8,B), $4.82\left(\mathrm{~d}, J=10.4 \mathrm{~Hz}, 1 \mathrm{H}, 11 \mathrm{~b}^{\mathrm{A}}\right), 4.77\left(\mathrm{~d}, J=13.1 \mathrm{~Hz}, 1 \mathrm{H}, 6^{\mathrm{B}}\right), 4.65(\mathrm{~d}, J=10.6 \mathrm{~Hz}, 1 \mathrm{H}$, $\left.11 b^{\mathrm{B}}\right), 4.52-4.34\left(\mathrm{~m}, 5 \mathrm{H}, 1^{\mathrm{A}, \mathrm{B}}, 3^{\mathrm{A}, \mathrm{B}}, 6^{\mathrm{A}}\right), 4.08\left(\mathrm{~d}, J=17.2 \mathrm{~Hz}, 1 \mathrm{H}, 3^{\mathrm{B}}\right), 3.71(\mathrm{~d}, J=17.7 \mathrm{~Hz}$, $\left.1 \mathrm{H}, 3^{\mathrm{A}}\right), 3.26\left(\mathrm{~d}, J=12.9 \mathrm{~Hz}, 1 \mathrm{H}, 1^{\mathrm{A}}\right), 2.88-2.78\left(\mathrm{~m}, 4 \mathrm{H}, 1^{\mathrm{A}, \mathrm{B}}, 6^{\mathrm{A}, \mathrm{B}}\right), 2.75-2.69\left(\mathrm{~m}, 2 \mathrm{H}, 7^{\mathrm{A}, \mathrm{B}}\right)$, 2.74-2.70 ( $\left.\mathrm{m}, 2 \mathrm{H}, 7^{\mathrm{A}, \mathrm{B}}\right), 2.60-2.58\left(\mathrm{~m}, 2 \mathrm{H}, 1^{\prime} \mathrm{A}, \mathrm{B}\right), 1.80-1.58\left(\mathrm{~m}, 8 \mathrm{H}, 2^{\prime} \mathrm{A}, \mathrm{B}, 3^{\prime} \mathrm{A}, \mathrm{B}, 5^{\prime} \mathrm{A}, \mathrm{B}, 6^{\mathrm{A}, \mathrm{B}}\right)$,

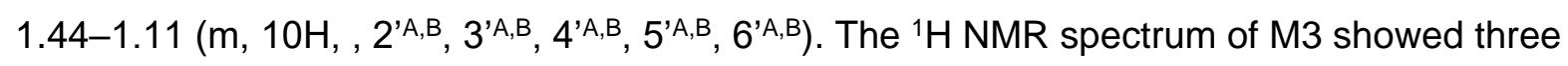
aromatic proton signals (one singlet at $\delta 6.58$ and two duplets at $\delta 7.25$ and $\delta 6.72-6.64$ ). The coupling pattern indicated a substitution of one benzene hydrogen with a hydroxyl group at either position 9 or 10 . ROESY cross-correlations of $\mathrm{H}-7$ to $\mathrm{H}-8$ and $\mathrm{H}-11 \mathrm{~b}$ to $\mathrm{H} 11$ were observable. Since $\mathrm{H}-8$ showed singlet multiplicity and $\mathrm{H}-11$ duplet multiplicity the substitution can be unambiguously assigned to position 9 (Figure 5, Supplemental Figure 23-27). 


\section{Discussion}

In summary, we were able to elucidate the absolute structure of three known and two unknown hydroxy metabolites of S-PZQ using the CS-XRD. UPLC-qTOF analysis and target-structure prediction of the fragmentation pattern allowed us to determine the type of metabolism and to limit the position of hydroxylation to a certain part of the molecule. However, only the crystallographic data could give information about the stereochemistry and pinpoint the exact position of the inserted hydroxy groups at the 4'-C position of the cyclohexane moiety (M1 and M2) as well as the 7-C (M4), 9-C (M3) and 11b-C (M6) positions at the core moiety. The structure of metabolite M3 was furthermore confirmed by NMR analysis.

The large number of different metabolites and impurities from the in vitro incubation matrix (e.g., buffer salts, cofactor, microsomal stock solution) required a lengthy semi-preparative sample separation and purification process as well as the selection of different columns. Metabolite M3, M4 and M5 were coeluting in the first chromatographic purification step. The application of a second column allowed the separation of M4, which could be successfully identified as S-7-hydroxy S-PZQ. First experiments of the second peak using the CS-XRD were unsuccessful, only diffuse electron density could be observed in the CS pores, indicating successful guest inclusion, but no regular order of analyte molecules, which is a necessary requirement for the assignment of guest compounds. From our experience, additional impurities in analyte material investigated with the CS-XRD, could lead to such behavior. Therefore, a third chromatographic step was conducted for further purification, which allowed the detection of an additional metabolite and thus the separation of M3 and M5. CS-XRD analysis of $\mathrm{M} 3$ allowed to identify the position of hydroxylation at 9-C of the aromatic ring. The crystallographic analysis of M5 however did not reveal the structure of the metabolite as a guest molecule in the CS pores, but MS and UV-VIS data strongly indicated that the hydroxylation occurred at the aromatic benzene ring. The structure of metabolite M6, which is known from literature, was confirmed and the presence of both enantiomers in vitro was shown. The analysis of both enantiomers as a racemic mixture and individually after chiral separation 
allowed the determination of the respective configuration as $S$-11b-hydroxy PZQ and $R-11 \mathrm{~b}$ hydroxy PZQ.

The application of the CS-XRD for S-PZQ and its metabolites demonstrates that this technology can be applied for metabolite identification studies in drug discovery where metabolites are only available in trace amounts from in vitro incubation. In comparison to the CS analysis, a high amount of substance was needed to conduct the NMR spectrum of M3, prolonging the lengthy sample purification process from days to weeks to collect sufficient analyte material. Furthermore, no information of the absolute configuration was obtained. The low abundancy of metabolite M4 in the incubation solution only allowed the chromatographic preparation of 1-2 $\mu \mathrm{g}$ of sample material, which is insufficient for complete structure analysis by NMR. However, the CS-XRD could be applied and allowed the successful determination of M4.

Our results show that the CS-XRD in combination with MS data is a valuable tool for absolute structure identification of metabolites in early phases of drug discovery and development, it constitutes a powerful tool for human metabolite identification from in vitro incubations and allows earlier risk mitigations associated with metabolites in safety testing guidelines (FDA, 2016). The technology was able to provide new knowledge of the metabolism of $P Z Q$, which has been used for the treatment of schistosomiasis for decades. The structural information at the atomic level were gained using only trace amounts of analyte material, outlining a significant advantage in comparison to other techniques like MS or NMR. Thus, we successfully confirmed the structure of three known hydroxy metabolites and elucidated the complete molecular scaffold of two unknown metabolites of S-PZQ.

To fully benefit from this technology, further investigation of the CS method is still needed to expand the chemical space of possible guest analytes by applying new types of CS with different binding sites and pore sizes to successfully encapsulate a wide range of target analytes in the CS pores and thus to successfully elucidate their complete structure. 


\section{Acknowledgements}

The authors gratefully thank Ralf-Erwin Licht for his excellent experimental contribution on metabolite profiling. We also acknowledge Holger Scheible, Dominique Perrin, Hanno Schieferstein and Benedikt Lang for useful discussions and support of this study. 


\section{Authorship Contributions}

Participated in research design: Rosenberger, Badolo

Conducted experiments: Rosenberger

Contributed new reagents or analytic tools: Kühn, Georgi

Performed data analysis: Rosenberger, Jenniches, Marx

Wrote or contributed to the writing of the manuscript: Rosenberger, Jenniches, von Essen,

Khutia, Kühn, Marx, Hirsch, Hartmann, Badolo 


\section{References}

Biradha K, and Fujita M (2002) A springlike 3D-coordination network that shrinks or swells in a crystal-to-crystal manner upon guest removal or readsorption. Angew Chemie - Int Ed 41:3392-3395.

Brunet G, Safin DA, Aghaji MZ, Robeyns K, Korobkov I, Woo TK, and Murugesu M (2017) Stepwise crystallographic visualization of dynamic guest binding in a nanoporous framework. Chem Sci 8:3171-3177.

Dolomanov O V, Bourhis LJ, Gildea RJ, Howard JAK, and Puschmann H (2009) OLEX2 : a complete structure solution, refinement and analysis program. J Appl Crystallogr 42:339-341, International Union of Crystallography.

FDA (2016) Safety Testing of Drug Metabolites - Guidance for Industry, US Department of Health and Human Services FDA Center for Drug Evaluation and Research, Silver Spring, MD.

Holčapek M, Jirásko R, and Lísa M (2010) Basic rules for the interpretation of atmospheric pressure ionization mass spectra of small molecules. J Chromatogr A 1217:3908-3921.

Hoshino M, Khutia A, Xing H, Inokuma Y, and Fujita M (2016) The crystalline sponge method updated. Acta Crystallogr Sect B 3:139-151.

Huang J, Bathena SPR, and Alnouti Y (2010) Metabolite Profiling of Praziquantel and its Analogs During the Analysis of in vitro Metabolic Stability Using Information- Dependent Acquisition on a Hybrid Triple Quadrupole Linear Ion Trap Mass Spectrometer. Drug Metab Pharmacokinet 25:487-499.

Hübschle CB, Sheldrick GM, and Dittrich B (2011) ShelXle : a Qt graphical user interface for SHELXL. J Appl Crystallogr 44:1281-1284.

Inokuma Y, Ukegawa T, Hoshino M, and Fujita M (2016) Structure determination of microbial metabolites by the crystalline sponge method. Chem Sci 7:3910-3913.

Inokuma Y, Yoshioka S, Ariyoshi J, Arai T, Hitora Y, Takada K, Matsunaga S, Rissanen K, and Fujita M (2013) X-ray analysis on the nanogram to microgram scale using porous complexes. [published correction appears in Nature (2013) 495:461-466]. Nature $501: 262$

Kovač J, Vargas M, and Keiser J (2017) In vitro and in vivo activity of R- and S- praziquantel enantiomers and the main human metabolite trans-4-hydroxy-praziquantel against Schistosoma haematobium. Parasites and Vectors 10.

Lerch C, and Blaschke G (1998) Investigation of the stereoselective metabolism of praziquantel after incubation with rat liver microsomes by capillary electrophoresis and liquid chromatography-mass spectrometry. J Chromatogr B Biomed App/ 708:267-275.

Meister I, Ingram-Sieber K, Cowan N, Todd M, Robertson MN, Meli C, Patra M, Gasser G, and Keiser J (2014) Activity of praziquantel enantiomers and main metabolites against Schistosoma mansoni. Antimicrob Agents Chemother 58:5466-5472.

Nleya L, Thelingwani R, Li X, Cavallin E, Isin E, and Nhachi C (2019) The effect of ketoconazole on praziquantel pharmacokinetics and the role of CYP3A4 in the formation of X-OH-praziquantel and not 4-OH-praziquantel. Eur J Clin Pharmacol 75:1077-1087.

Park SK, Friedrich L, Yahya NA, Rohr C, Chulkov EG, Maillard D, Rippmann F, Spangenberg T, Marchant JS (2021) Mechanism of praziquantel action at a parasitic flatworm ion channel. Sci.Transl.Med 13: eabj5832

Ramadhar TR, Zheng SL, Chen YS, and Clardy J (2015) Analysis of rapidly synthesized guest-filled porous complexes with synchrotron radiation: Practical guidelines for the crystalline sponge method. Acta Crystallogr Sect A Found Crystallogr 71:46-58.

Ramanathan R, Su AD, Alvarez N, Blumenkrantz N, Chowdhury SK, Alton K, and Patrick J (2000) Liquid chromatography/mass spectrometry methods for distinguishing $\mathrm{N}$ - oxides from hydroxylated compounds. Anal Chem 72:1352-1359.

Rosenberger L, Essen C Von, Khutia A, Kühn C, Georgi K, Hirsch AKH, Hartmann RW, and Badolo $L$ (2021) Crystalline sponge affinity screening: A fast tool for soaking condition optimization without the need of X-ray diffraction analysis. Eur J Pharm Sci 164:105884.

Rosenberger L, Von Essen C, Khutia A, Kühn C, Urbahns K, Georgi K, Hartmann RW, and Badolo L (2020) Crystalline sponges as a sensitive and fast method for metabolite 
identification: Application to gemfibrozil and its phase I and II metabolites. Drug Metab Dispos 48:587-593.

Sakurai F, Khutia A, Kikuchi T, and Fujita M (2017) X-ray Structure Analysis of N-Containing Nucleophilic Compounds by the Crystalline Sponge Method. Chem - Eur J 23:1503515040.

Schepmann D, and Blaschke G (2001) Isolation and identification of 8-hydroxypraziquantel as a metabolite of the antischistosomal drug praziquantel. $J$ Pharm Biomed Anal 26:791-799.

Sheldrick GM (2015) Crystal structure refinement with SHELXL. Acta Crystallogr Sect C C71:3-8.

Vendrell-Navarro G, Scheible $H$, Lignet $F$, Burt $H$, Luepfert C, Marx A, Abla N, Swart $P$, and Perrin D (2020) Insights into Praziquantel Metabolism and Potential Enantiomeric Cytochrome P450-Mediated Drug-Drug Interaction. Drug Metab Dispos 48:481-490.

Wang H, Fang ZZ, Zheng Y, Zhou K, Hu C, Krausz KW, Sun D, Idle JR, and Gonzalez FJ (2014) Metabolic profiling of praziquantel enantiomers. Biochem Pharmacol 90:166178.

World Health Organiziation (2011) Weekly epidemiological record. No. 9, 86:73-80.

World Health Organiziation (2020) Schistosomiasis fact sheet. https://www.who.int/newsroom/fact-sheets/detail/schistosomiasis. 


\section{Footnotes}

- This work received no external funding.

- Conflict of interest: The authors declare a conflict of interest. L.R., J.J., C.v.E., A.K., C.K., A.M., K.G. and L.B. are employees of Merck KGaA, Darmstadt, Germany. 


\section{Figure Legends}

Figure 1. Chemical structure of $S$-praziquantel

Figure 2. MS/MS spectra and proposed structures of S-PZQ and its metabolites M1-M6

Figure 3. Full-scan LC-MS chromatograms of $S-P Z Q$ incubated with HLM for $24 \mathrm{~h}$ in the presence of NADPH as cofactor on a Lux Cellulose-2 column (A) and PZQ metabolites M3M6 after semi-preparative HPLC separation and fractionation (B); M1, S-trans-4'-hydroxy PZQ; M2, S-cis-4' hydroxy PZQ, M3, S-9-hydroxy PZQ; M4, S-7-hydroxy S-PZQ; M5, still unknown; M6, S-11b-hydroxy PZQ and R-11b-hydroxy PZQ.

Figure 4. (A) Asymmetric unit of $\left[\left(Z n l_{2}\right)_{3}(\mathrm{tpt})_{2}\right]_{n}$ with one S-9-hydroxy PZQ (M3) and two cyclohexane molecules. The crystal structure exhibits $\mathrm{C}-\mathrm{H} \cdots \mathrm{O}$ (red dashed line) interactions between the hydroxy metabolite and the CS framework. (B) Electron density map $F_{\mathrm{o}}$ [contoured

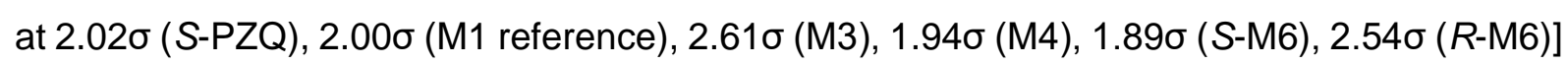
of the complete molecule of S-PZQ and M1, as well as the core moiety of the incubation samples M3, M4, S-M6 and R-M6.

Figure 5. $700 \mathrm{MHz}{ }^{1} \mathrm{H}$ NMR spectrum of S-9-hydroxy PZQ (M3) 
Figure 1

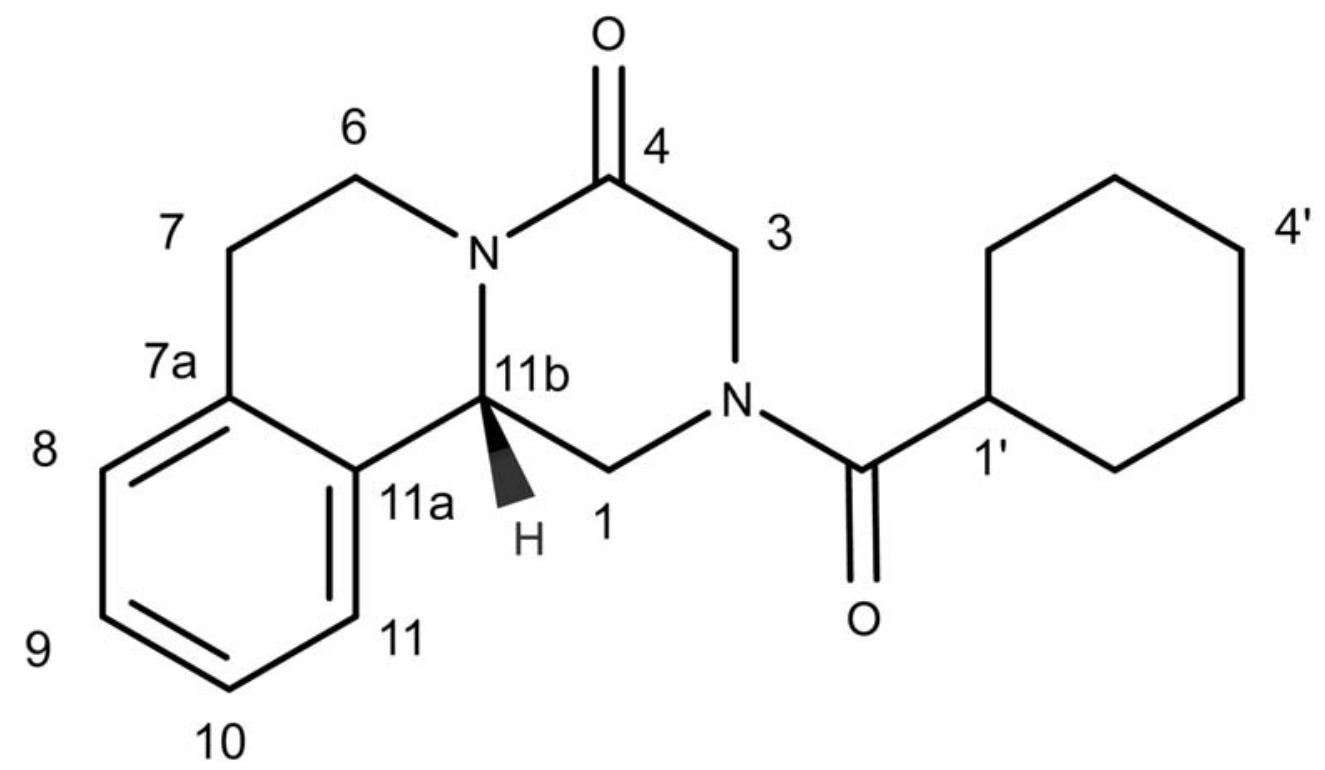

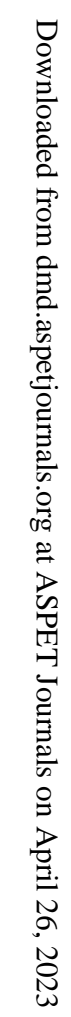


Figure 3

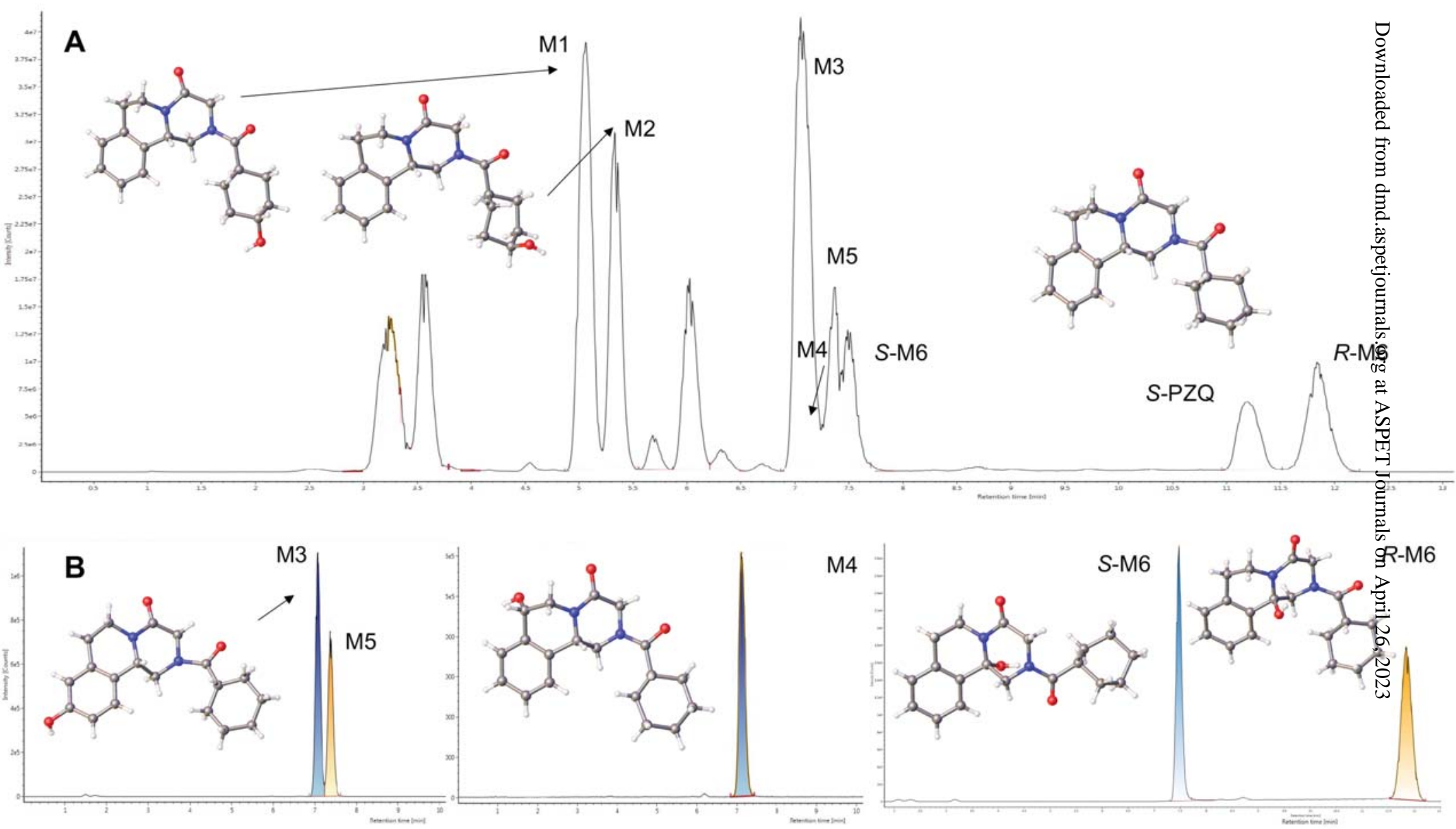


Figure 4

A

B
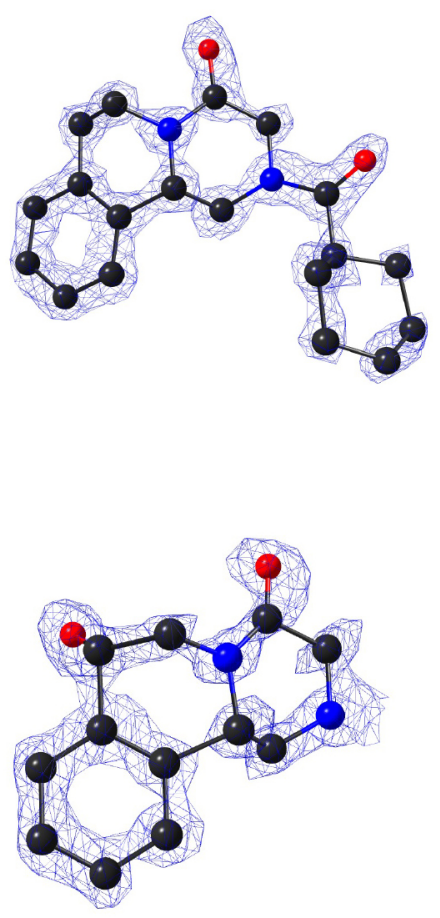

M4

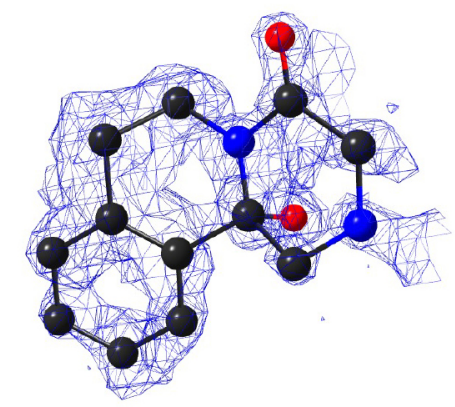

S-M6

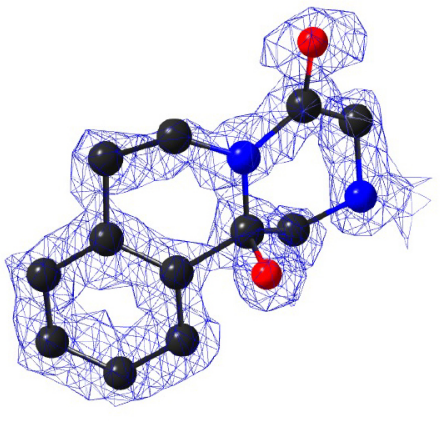

R-M6 
Figure 5

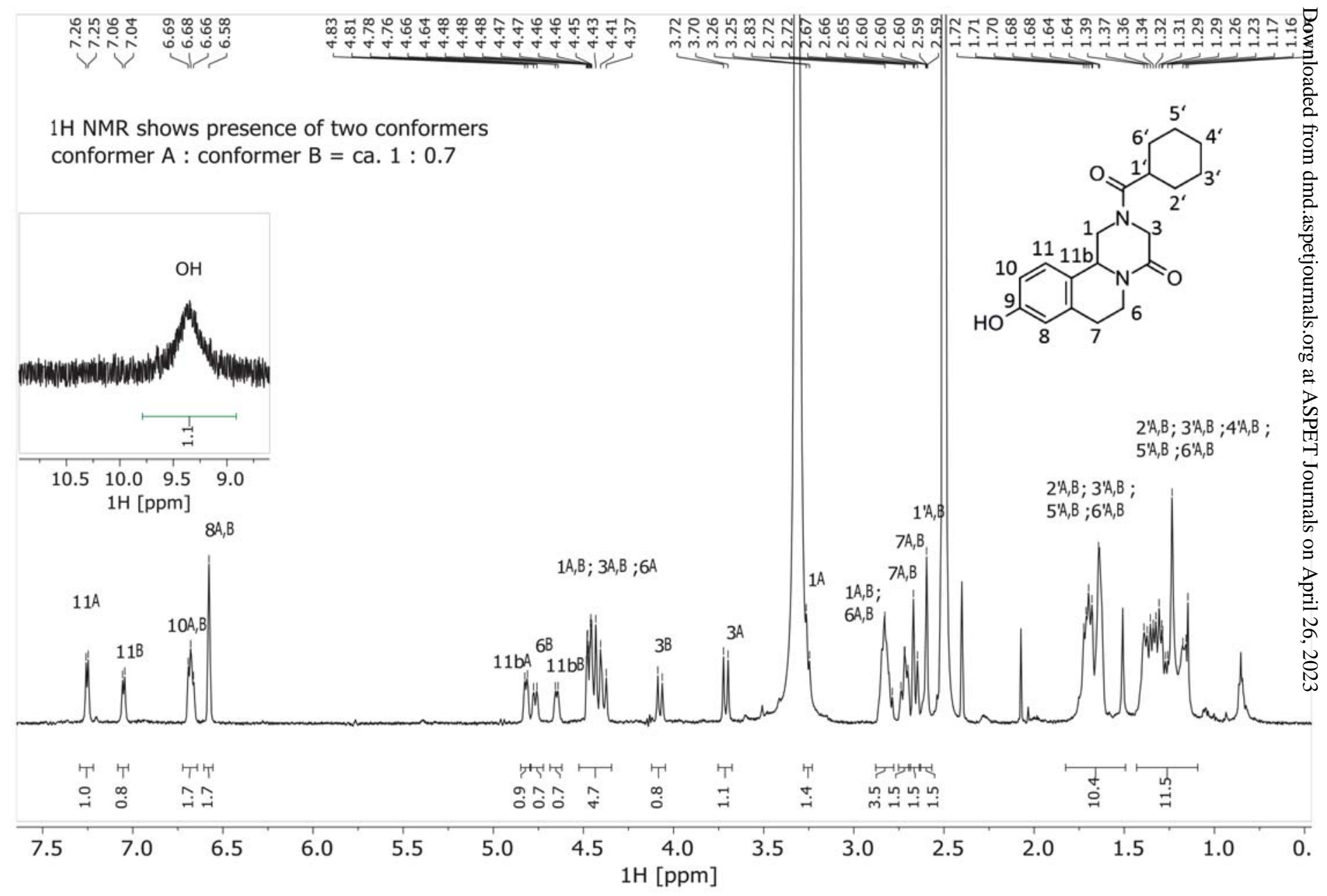

